\title{
Technique for reconstructing a surface shape for measuring coordinates
}

\author{
V. Kh. SaĬnov, ${ }^{\text {a) }}$ Zh. I. Kharizanova, and E. V. Stoĭkova \\ Central Laboratory of Optical Recording and Data Processing, Sofia, Bulgaria
}

Kh. M. Ozaktas and L. Onural

Bilkent University, Ankara, Turkey

(Submitted December 27, 2005)

Opticheskiü Zhurnal 73, 49-54 (July 2006)

\begin{abstract}
This paper describes a method of projecting interference fringes as one of the most accessible techniques for measuring the coordinates of objects and scenes that can be used when solving inverse problems in dynamic holographic display, where the coordinates need to be measured in order to compute diffraction structures when reconstructing three-dimensional images. A comparative analysis is presented of the experimental results obtained with successive projections of interference patterns with two different periods, using a Mach-Zehnder interferometer in coherent light and a micromirror projector with digital generation of fringes in white light. The use of the method is limited by the size of the objects and scenes. The possibilities of using more refined methods, including the holographic approach to phase reconstruction, are discussed.

(C) 2006 Optical Society of America.
\end{abstract}

\section{PHASE RECONSTRUCTION FOR MEASURING ABSOLUTE COORDINATES}

Accurate information on the coordinates of objects and three-dimensional scenes is needed in order to implement a dynamic holographic display. The main requirement in this case is to make measurements of a random distribution of objects in a scene in real time. Optical profilometry methods offer the most promising solution of this problem. Some of the optical systems that exist and are for sale for measuring the coordinates of actual objects have been developed on the basis of laser scanning and triangulation. ${ }^{1,2}$ Because the surface is scanned in a single direction "point to point" with a limited information-readout rate, the state of the surroundings (vibrations, air turbulence, etc.) has a strong effect on the measurement accuracy, especially in large-scale scenes under actual conditions. Special attention should be paid to the wide class of phase-reconstruction methods based on moiré and projection interferometry. ${ }^{3,4}$ The main advantage of these methods is the simplicity, high accuracy, and rate of the measurements.

Information on the surface of an object can be obtained by reconstructing the phase of an object wave front and by recording the intensities in spatial and frequency domains. Multiplicity in spatial frequencies in profilometry based on the Fourier transformation, in combination with a complicated phase-reconstruction algorithm, provides measurement in real time by a single recording, and this is very essential when measuring moving objects isolated in space. ${ }^{5}$ A disadvantage of this method is the comparatively low measurement accuracy, which limits its wide practical use. From this viewpoint, the technique of projecting interference fringes with the introduction of a phase shift ${ }^{4}$ is more suitable for measuring and reconstructing three-dimensional scenes. This simplified approach is widely used in optical metrology for surface measurement and nondestructive testing. ${ }^{6-9}$ The fringes can be projected with coherent light, using, for ex- ample, a Michelson interferometer, or in incoherent light by means of two-coordinate spatial light modulators, such as liquid-crystal or micromirror projectors. The recording of fringes deformed by an object's surface for a given phase shift $\phi$ makes it possible to reconstruct the phase $\Delta \varphi$ modulated by the object, using the interferogram:

$$
I(x, y)=\bar{I}(x, y)\{1+\eta(x, y) \cos [\Delta \varphi(x, y)+\phi]\},
$$

where $I(x, y)$ is the intensity at point $(x, y), \bar{I}(x, y)$ is the averaged intensity, and $\eta$ is the contrast of the interference fringes. At least three independent measurements are needed for this purpose, to determine the three unknown parameters in Eq. (1). Various phase-shift algorithms have been developed. ${ }^{4,10,11}$ A popular five-step algorithm is based on five independent measurements of the intensity $I_{i}$ corresponding to the five phase shifts $\phi_{i}=(i-3) \pi / 2$, where $i=1-5$, as a result of which the calibration error is decreased:

$$
\Delta \varphi=\tan ^{-1}\left[2\left(I_{2}-I_{4}\right) /\left(2 I_{3}-I_{5}-I_{1}\right)\right] .
$$

The illuminating system has a substantial effect on the quality of the measurements. The advantages of interferometrically generated fringes are that they increase the illumination efficiency, give a fringe contrast that is high and unrestricted in depth of the scene, and provide more flexible formation of the fringes. The fringes possess a pure sinusoidal profile, and this provides high measurement accuracy. The effect of the external lighting under working conditions can be eliminated by using an appropriate interference filter. Liquid-crystal projectors with incoherent light and digital generation of fringes of various frequencies are characterized by high response rate but are limited in resolution. Plasma and micromirror devices create higher irradiance brightness. The best contrast is inherent to micromirror modulators, and this, along with high optical efficiency, makes them more promising for practical applications. The nonsinusoidal nature of the fringe profile because of the limited number and 


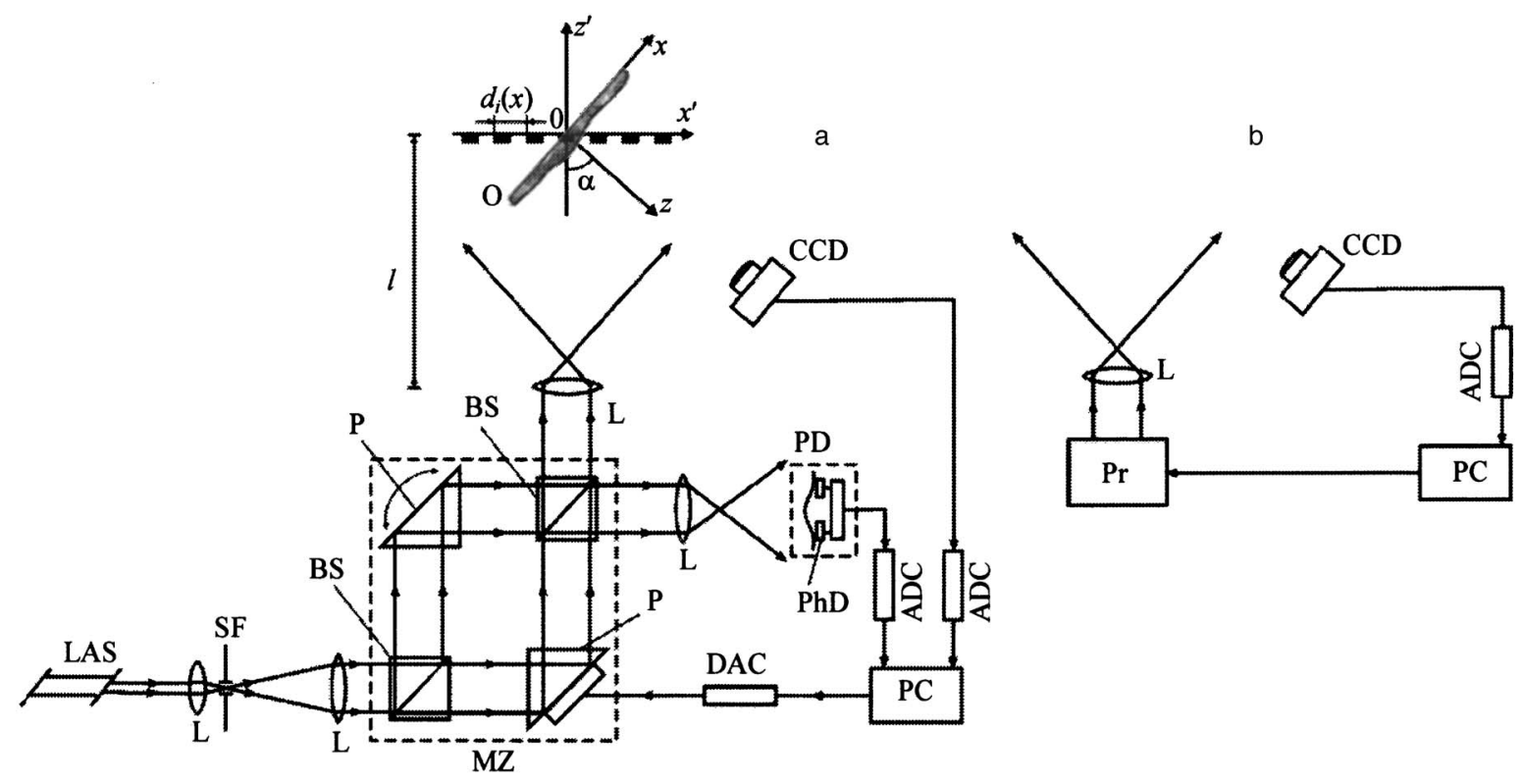

FIG. 1. Experimental apparatus for projecting fringes in coherent (a) and white (b) light. LAS is a laser, O is the object, L is a lens, BS is a beamsplitter, SF is a spatial filter, $\mathrm{P}$ is a prism, MZ is a Mach-Zehnder interferometer, PD is a photodetector, PhD are photodiodes, $\mathrm{PC}$ is a computer, ADC is an analog-todigital converter, CCD is a CCD camera, Pr is a micromirror projector, and DAC is a digital-to-analog converter.

size of the pixels is the main drawback of the indicated projection systems. Moreover, defocusing in areas where the relief is of great depth reduces the fringe contrast and the measurement accuracy.

As follows from Eq. (2), the phase differences are not determined unambiguously, but to within $2 \pi$, and this requires joining the phase values at the break points caused by the tangential dependence. To correctly join them, an estimate of the quality of the phase data should be introduced in order to distinguish the break points and zones inherent to the object itself from those caused by noise, vibrations, shadowing, etc. Good estimates of the quality of the individual pixels in the phase pattern can be obtained on the basis of the variance of the partial derivatives of the phase with respect to spatial coordinates $x$ and $y$ or the maximum value of the phase gradient, as well as by combining them. ${ }^{11}$ The maximum phase gradient is determined in such a way that it exceeded the maximum value of the partial derivatives of the phase for each pixel, where the maximum values are estimated by averaging within a $k \times k$ window for each pixel. In this paper, the joining of the phase is done using a method based on tracing the phase trajectory, for which, if there are no break points, the phase can be integrated along any trajectory.

\section{MEASURING THE THREE-DIMENSIONAL COORDINATES BY THE METHOD OF PROJECTION OF TWO-PERIOD INTERFERENCE PATTERNS}

Three-dimensional coordinates can be efficiently measured by projecting two-period interference patterns. ${ }^{12-14}$ This approach can be further improved by double symmetric illumination or double symmetric recording to reduce the influence of shading.

Figure 1 shows the layout for implementing a method of projecting bright fringes by means of a Mach-Zehnder interferometer (Fig. 1a) and a micromirror projector (Fig. 1b) with digital synthesis of the fringes. The method is based on the generation in the $\left(x^{\prime}, y^{\prime}, 0\right)$ plane of interference fringes with periods $d_{1}$ and $d_{2}$ that are parallel to the $Y^{\prime}$ axis. The $Y$ and $Y^{\prime}$ axes are perpendicular to the plane of the drawing. The phase of the projected fringes is determined as $\varphi_{i}^{\prime}$ $=2 \pi x^{\prime} / d_{i}, i=1,2$. The phase is reconstructed in the $X Y Z$ coordinate system, with the $Z$ axis oriented parallel to the optical axis of the solid-state (CCD) recording camera. Angle $\alpha$ is the slope angle of illumination axis $Z^{\prime}$ with respect to observation axis $Z$. Phase $\Delta \varphi_{i}(x, y)$ in the intensity distribution given by Eq. (1) is determined by means of the five-step algorithm given in Eq. (2) for each fringe pattern corresponding to a given period. The smaller period is chosen, allowing ten pixels per period, which in essence exceeds the theoretical requirement for accurately measuring the phase. The phase $\Delta \varphi_{i}(x, y)$ can be represented in the $X Y Z$ coordinate system as

$$
\begin{aligned}
\Delta \varphi_{i}(x, y)= & \varphi_{i}(x, y)-\varphi_{0}=\left\{2 \pi[l x \cos \alpha+l z(x, y) \sin \alpha] / d_{i}[l\right. \\
& -z(x, y) \cos \alpha+x \sin \alpha]\}-\varphi_{0},
\end{aligned}
$$

where $i=1,2 ; z(x, y)$ is the relief of the object at point $(x, y)$, $l$ is the distance from the object to the exit pupil of the illumination objective, and $\varphi_{0}$ is an unknown calibration constant. Subtracting phase distributions $\Delta \varphi_{i}(x, y)$ and assuming that

$$
\left[\varphi_{2}(x, y)-\varphi_{1}(x, y)\right] / 2 \pi \equiv N_{x, y},
$$

where $N_{x y}$ are numbers obtained on the basis of the measured phase values, we obtain the expression for coordinate $z$ in the form 


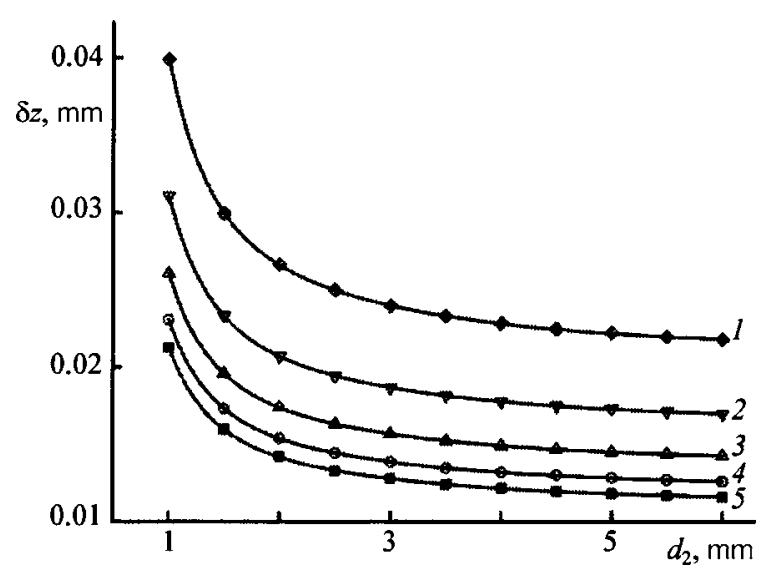

FIG. 2. Measurement indeterminacy $|\delta z|$ vs period $d_{2} . \alpha=30^{\circ}(1), 40^{\circ}(2)$, $50^{\circ}(3), 60^{\circ}(4), 70^{\circ}(5) . d_{1}=0.5 \mathrm{~mm}, x=0, \delta N=0.02, l=2000 \mathrm{~mm}, N=1.5$.

$$
\begin{aligned}
z(x, y)= & {\left[N_{x y}(l+x \sin \alpha)+\frac{d_{2}-d_{1}}{d_{1} d_{2}} l x \cos \alpha\right] / } \\
& {\left[N_{x y} \cos \alpha-\frac{d_{2}-d_{1}}{d_{1} d_{2}} l \sin \alpha\right] }
\end{aligned}
$$

The method's sensitivity mainly depends on the accuracy with which the phase difference is measured, i.e., on the accuracy with which $N_{x y}$ is determined. The influence of inaccuracy in determining $l$ and $\alpha$ can be neglected.

Based on Eq. (5), the sensitivity of the method, determined by the minimum measurable difference of the $z$ coordinates, can be written as

$$
\begin{aligned}
\delta z= & {\left[d_{1} d_{2}\left(d_{1}-d_{2}\right) /(x+l \sin \alpha) \delta N_{x y}\right] /\left[d_{1} d_{2} N_{x y} \cos \alpha\right.} \\
& \left.+\left(d_{1}-d_{2}\right) / \sin \alpha\right)^{2} .
\end{aligned}
$$

The measurement accuracy increases with increasing difference of the fringe periods $d_{1}-d_{2}$ and with illumination angle $\alpha$ but is not identical over the length of the object and decreases as its transverse size increases. Figure 2 shows the measurement indeterminacy $|\delta z|$ for a set of illumination angles $\alpha$ as a function of period $d_{2}$ at fixed values of $d_{1}$ $=0.5 \mathrm{~mm}, \delta N_{x y}=0.02$, and $N_{x y}=1.5$.

The results of measuring the surface by the projection of two-period interference fringes (Fig. 1a) at $\alpha=70^{\circ}, l$ $=1500 \mathrm{~mm}, d_{1}=0.5 \mathrm{~mm}$, and $d_{2}=2 \mathrm{~mm}$ are shown in Fig. 3 . The object of measurement was a cylindrical vessel made from a composite material with an outer diameter of $200 \mathrm{~mm}$ under a pressure of $500 \mathrm{kP}$. The deformation of part of the side wall of the cylinder with dimension $100 \times 100 \mathrm{~mm}$ was measured. Figure 3 a contains segments of two-period fringes modulated by the surface of the object. The reconstructed phase difference in the intensity scale of white light modulo $2 \pi$ is shown in Fig. 3b. The corresponding joined image of the phase difference and a three-dimensional image are shown in Figs. 3c and 3d. The sensitivity of the method makes it possible to record the inhomogeneous deformation of the object's surface under pressure.

Comparative experiments of the use of two-period projection interferometry with generation of fringes by means of a Mach-Zehnder interferometer (Fig. 1a) and a micromirror

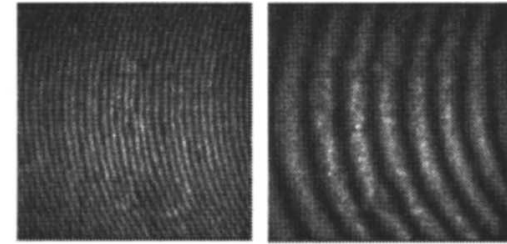

b

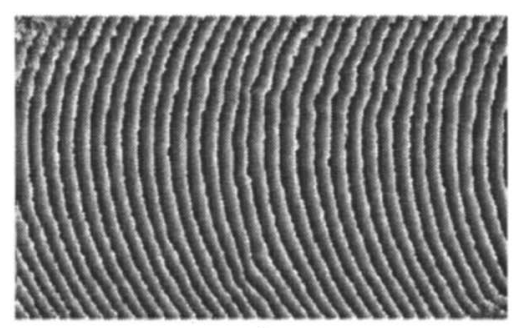

c
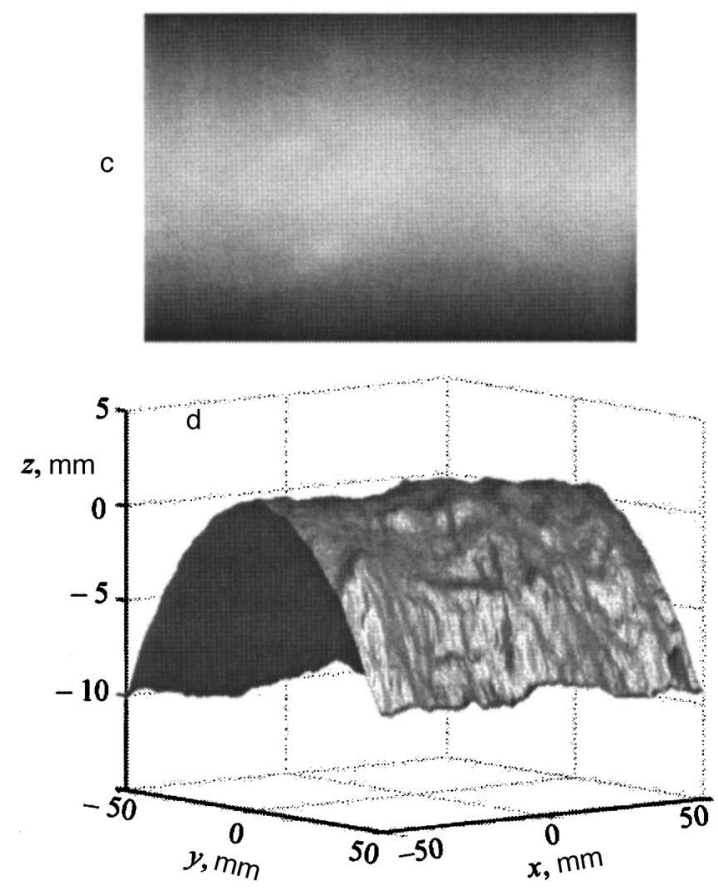

FIG. 3. Measurement of the absolute coordinates of the central part of a vessel made from a composite material using projection two-period interferometry. (a) Segments of projected fringes with periods $d_{1}$ and $d_{2}$, (b) phase image modulo $2 \pi$, (c) reconstructed phase image, (d) three-dimensional image of the surface.

projector with digital synthesis (Fig. 1b) were made on the same object-a $30 \times 30 \times 30-\mathrm{mm}$ cube. ${ }^{15}$ In both experiments, the fringe periods were $d_{1}=17.1$ and $d_{2}=8.5 \mathrm{~mm}, \alpha$ $=30^{\circ}$, and $l=900 \mathrm{~mm}$. The reconstructed phase difference and the corresponding joined images in a white-light intensity scale are shown in Figs. 4a and 4cwith generation of fringes in coherent light by a Mach-Zehnder interferometer and in Figs. $4 \mathrm{~b}$ and $4 \mathrm{~d}$ with digital generation of fringes by a micromirror projector in white light. It should be pointed out that the sensitivity and measurement accuracy in the given experiments are substantially worse than the results shown in Fig. 3, because of the smaller illumination angle $\alpha$ and the impossibility of making the optimum choice of the periods of the interference fringes. This is associated with the limited resolution and the small size of the modulator of the micromirror projector $(800 \times 600$ pixels $)$ for creating fringes with 

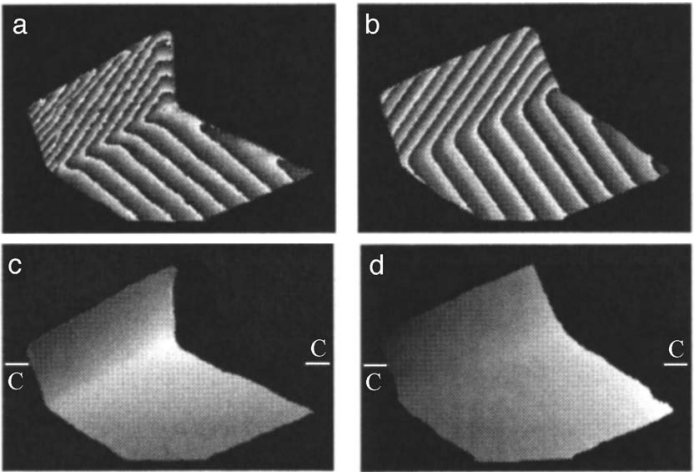

FIG. 4. Experimental results of the measurement of a test object by the method of fringe projection in coherent light (a and c) and in white light (b and $\mathrm{d})$. ( $\mathrm{a}$ and $\mathrm{b})$ Phase image, (c and d) reconstructed phase image.

a sinusoidal profile. A comparison of the results is shown in Fig. 5 for the points of intersection along line $\mathrm{C}-\mathrm{C}$, indicated in Figs. 4c and 4d. As expected, the profile-measurement accuracy by the method of interferometrically generated fringes is somewhat greater, but the method of projecting the fringes in white light is simpler and more convenient when solving a number of practical problems. To make measurements in real time by the fringe-projection method, it is necessary to illuminate the object simultaneously with phaseshifted fringes that are generated at different wavelengths, using interference beamsplitters during the recording.

\section{THE HOLOGRAPHIC APPROACH TO PHASE RECONSTRUCTION}

The method of interference-fringe projection presented here can be used to solve inverse problems in a dynamic holographic display, since the coordinates of the objects and scenes need to be measured in order to compute the diffraction structures when reconstructing three-dimensional images. The use of the method is limited mainly by the size of the objects and scenes, and this requires further improvement of the approaches when reconstructing the phase. Many phase-reconstruction methods have been proposed, based on

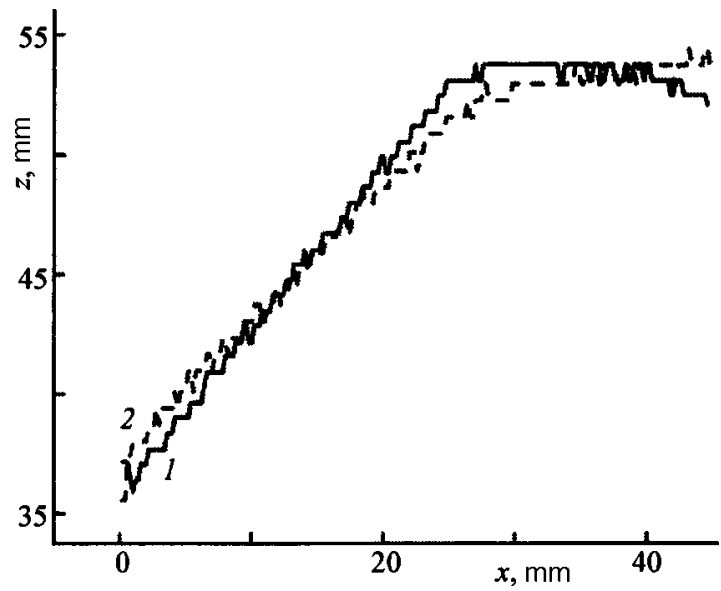

FIG. 5. Comparison of cross sections indicated by line $\mathrm{C}-\mathrm{C}$ in Figs. $4 \mathrm{c}$ and 4d. 1-Method of interferometrically generated fringes, 2-method of fringe projection.

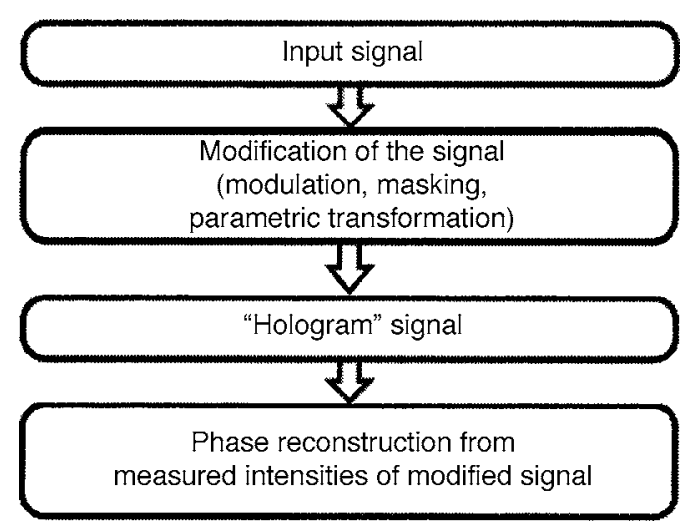

FIG. 6. Diagram of solution of the coordinate-measurement problem.

independent intensity measurements ${ }^{16,17}$ of the wave front in the plane of the spatial coordinates (for example, in the image plane) and in the frequency plane. It is especially promising to use the fractional Fourier transformation, as a generalization of this approach to the case of multiple fractional Fourier transformations. Phase reconstruction only on the basis of the Fourier transformation has also been investigated, with additional limitations with respect to size, nonnegativity, etc. The possibilities of using multiple intensity measurements with a given coding (modulation) of the input signal have been studied to a lesser degree. For this purpose, it is also possible to introduce or to use variations of the input signal introduced by the object. In fact, most phasereconstruction methods are based on intensity measurements with two independent representations of the signal for comparison of independent equations, which are solved iteratively and usually require additional analysis.

In principle, with all phase-reconstruction methods, a light-intensity measurement is carried out for which information on the amplitude and phase of the wave front can be obtained, and this is characteristic of holography. However, the obvious connection of holography with phasereconstruction methods has been indicated comparatively recently. A set of signals obtained by a given phasereconstruction algorithm from measured intensities, with the introduction of additional limitations, can be taken in practice for a complete description or "hologram" of the signal. The most general description of the signal is the image intensity, transformed or modified by some method. Thus, the recorded intensity and its Fourier representation or any of the fractional Fourier representations, taken together, constitute the hologram of the signal. Other sets of data can be constituted by modulating, masking, or other suitable modification of the input signal. The intensities created by this modified input signal subsequently make it possible to reconstruct the phase and in this sense constitute a hologram of the signal. Based on these considerations, the solution of the problem of measuring the coordinates can reduce to comparing a set of modified images, as schematically shown in Fig. 6. In principle, there are many forms of modifications, including shift, slope, modulation, masking, and parametric transformation of the signal, for making independent measurements of the intensities and reconstructing the phase. 
This work was carried out under Contract 511568 "3DTV" at part of FP6-EC.

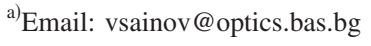

${ }^{1}$ F. Chen, G. M. Brown, and M. Song, "Overview of three-dimensional shape measurement using optical methods," Opt. Eng. (Bellingham) 39, 10 (2000).

${ }^{2}$ F. Blais, "Review of 20 years of range sensor development," J. Electron. Imaging 13, No. 1, 231 (2004).

${ }^{3}$ P. K. Rastogi, ed., Holographic Interferometry Principles and Methods (Springer Verlag, Berlin: 1994).

${ }^{4}$ T. Kreis, Handbook of Holographic Interferometry: Optical and Digital Methods (Wiley-VCH GmbH\&Co. KGaA, Weinheim, 2005).

${ }^{5}$ M. Takeda, Q. Gu, M. Kinoshita, H. Takai, and Y. Takahashi, "Frequencymultiplex Fourier-transform profilometry: a single-shot three-dimensional shape measurement of objects with large height discontinuities and/or surface isolations," Appl. Opt. 36, 5347 (1997).

${ }^{6}$ M. Lehmann, P. Jacqot, and M. Facchini, "Shape measurements on large surfaces by fringe projection," Exp. Tech. 23, 31 (1999).

R. Sitnik, M. Kujavinska, and J. Wonznicki, "Digital fringe projection system for large-volume $360 \mathrm{deg}$ shape measurement," Opt. Eng. (Bellingham) 41, 443 (2002).

${ }^{8}$ K. Körner, R. Windecker, M. Fleischer, and H. J. Tiziani, "One-grating projection for absolute three-dimensional profiling," Opt. Eng. (Bellingham) 40, 1653 (2001).

${ }^{9} \mathrm{C}$. Vincent, T. Fournel, and R. Fouquet, "Self-indexing of multiview fringe system,” Appl. Opt. 42, 1981 (2003).

${ }^{10}$ H. Liu, G. Lu, S. Wu, S. Yin, and F. Yu, "Speckle-induced phase error in laser-based phase-shifting projected fringe interferometry," J. Opt. Soc. Am. A 16, 1484 (1999).

${ }^{11}$ D. C. Ghiglia and M. D. Pritt, Two-Dimensional Phase Unwrapping (J. Wiley \& Sons, New York, 1998).

${ }^{12}$ V. Sainov, J. Harizanova, G. Stoilov, and P. Boone, "Relative and absolute coordinates measurement by phase-stepping laser interferometry," Optics and Lasers in Biomedicine and Culture 5, 50 (2000).

${ }^{13}$ V. Sainov, J. Harizanova, and A. Shulev, 'Two-wavelength and twospacing projection interferometry for real objects contouring," Proc. SPIE 5226, 184 (2003).

${ }^{14}$ J. Harizanova and V. Sainov, "Two-spacing projection phase-stepping interferometry for 3D-surface measurements of large-scale objects," Proc. SPIE 5478, 253 (2003).

${ }^{15} \mathrm{~J}$. Harizanova and A. Kolev, "Comparative study of fringes generation in two-spacing phase-shifting profilometry," Proc. SPIE 2005 [accepted for publication].

${ }^{16}$ M. Ertosun, H. Atli, H. M. Ozaktas, and B. Barshan, "Complex signal recovery from multiple fractional Fourier-transform intensities," Appl. Opt. 44, 4902 (2005).

${ }^{17}$ M. Ertosun, H. Atli, H. M. Ozaktas, and B. Barshan, "Complex signal recovery from two fractional Fourier-transform intensities: order and noise dependence," Opt. Commun. 244, 61 (2005). 